\title{
Survey on Food Recognition System Using Machine Learning
}

\author{
D.GaneshGaurav ${ }^{\mathrm{a}, 1}$, Parthasarathy $\mathrm{G}^{\mathrm{b}}$,Dhananja ${ }^{\mathrm{b}}$, Chiranthan $\mathrm{YC}^{\mathrm{b}}$, KomalBharti $^{\mathrm{b}}$ \\ ${ }^{a, b}$ School of Computing and IT, REVA University, Karnataka, India
}

\begin{abstract}
Food recognition system is essential framework in modern world, the motivations behind this recognition systems are assessing calorie and nutrients of food varieties and show the formula of the food. As all the processing on picture recognition is performed on a personal digital assistant (PDA), the framework doesn't have to send pictures to a server and runs solution to a normal advanced mobile phone in a constant one. Spontaneous image-based food acknowledgment is an isolated inspiring task. Conventional picture analysis preparing methodologies have fallen off with low order classification prediction in the past, while deep learning methodologies permitted the recognizable proof of food things. To recognize food items, a client ought to need to draw bounding boxes by contacting the screen first, and afterward the framework starts food thing acknowledgment inside the showed bounding boxes. Moreover, the framework assesses the direction of food areas with the higher SVM (Support Vector System) yield score is expected upon to be acquired, show it as an arrow on the screen to request that a client move an advanced mobile phone camera. This acknowledgment cycle is performed on and on about once each second. We will carry out this task as an Android advanced mobile phone application to utilize numerous CPU cores effectively for modern real-time food recognition system.
\end{abstract}

Keywords.Machine Learning, Image Analysis, SVM

\section{Introduction}

The new age Smartphone's have completely transformed the way we live, due to the improved digital payments technology people are looking for an easy and effective way to access information without wasting time. The outcome of this project will be that the user will simply have to scan the food and all the information, which is the food preparation method, the origin, calories count is displayed in a very informative way, this helps user to access all the information without actually searching the web which may not have an accurate data.

The appearance of deep learning innovation has upheld various intelligent zones by giving advanced techniques and strategies for improved food object prediction and recognition, in view of images object or videos. In software engineering, especially in computer vision application and artificial intelligence, image analysis is a basic task, with different new improvements upcoming from object recognition with deep learning techniques

${ }^{1}$ GaneshGaurav,School of Computing and IT, REVA University, Karnataka, India

E-mail id: d.ganeshgaurav@gmail.com, 


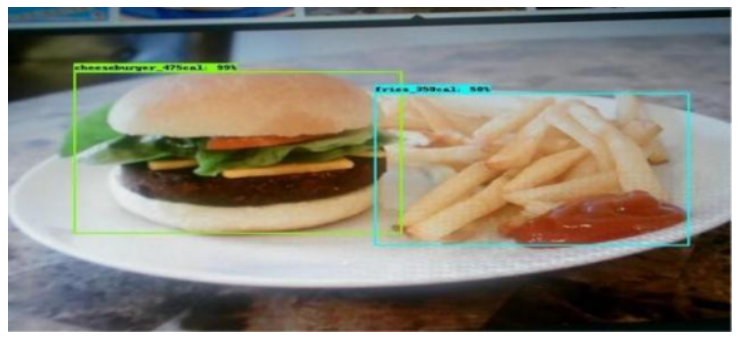

Figure 1.Recognising food items

In this paper we will carry out deep learning designs reliant on convolutional neural networks $(\mathrm{CNN})$ which are very productive in picture recognition system. These structures use a course of convolution layers and activation functions. The plan of the amount of layers and the amount of neurons in each layer, the choice of activation functions and training advancement calculation are indispensable. The GPU execution of $\mathrm{CNN}$ with include feature extractors proposed for building recognition, learned in a coordinated way and achieve commonly great results.
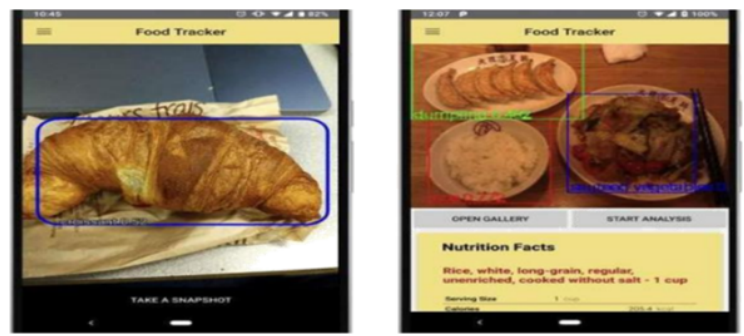

Figure 2.Detecting Multiple Food Items

\section{Literature Survey}

This paper is all about a smart application which is been discussed, with the help of mobile edge registering idea that empowers clients to evaluate and analyse their food consumption and backing nourishing dynamic. Mobile edge process to offload system calculations and correspondences to the edge is the methodology utilized, which surely help saving battery life, expanding the preparing limit, and improving client solace. In system creation we propose an a loosely coupled network for a savvy food scanner and afterward execute it utilizing different IoT sensors. Previously, the innovation patterns began to uncover the limits of clouds to host smart applications. A bunches of advancements in the space of Internet of Things (IoT), Big Data, and Deep learning supported the improvement of brilliant applications which for sure uncovered information ingestion as a significant clog of incorporated cloud solutions [1]. In the third paper they presented a study of a novel sensor system for real-time food substantiate. Smartphone videos, pattern recognition and image processing is being used by this sensor system. It has the capacity of Concurrent Version System (cvs.) that capture information of any food sample. The main aim is to replace human visual system in food substantiate with the help of high- level understanding, soft sensor of 
food substantiate through the accession, processing and analysis of digital images. The association between image attributes and quantitative data about food authenticity are used to determine the pattern, and hence new food samples are predicted [2]. In the fourth paper we have seen that the activity based on Smart watch can easily form basis of health applications and in biomedical, which includes applications that track user's eating habit[7-16]. The outcome of this paper accurately identify a large variety of activities in smart watches including hand-based and eating based activities that a smartphone cannot recognize effectively [3].

\section{Proposed System}

The existing model has a recognition system which recognizes the type of food (like e.g. Burger). The model which we are going to propose will recognize the food item (like e.g. Burger) and also shows its recipe and nutrition contents in that food item. In our proposed system for food picture classification, as a first step toward the improvement of a portable application, it provides dietary advice to diabetic and other patients by indicating the amount of calories, sugar content, carbs etc. in that food item. We will be developing the application in a form which the user must use this as a one stop destination for food information and this will eliminate the problems like false information's and time spent to search.

\section{Problem Definition}

The existing model has a recognition system which recognizes the type of food (like eg:Burger). The model which we are going to propose will recognize the food item (like eg: Burger) and also shows its name and its recipe. In our proposed system for food picture classification, as a first step toward the improvement of a portable application, it provides dietary advice to diabetic and other patients by indicating the amount of calories, sugar content, carbs etc. in that food item.

\section{System Design}

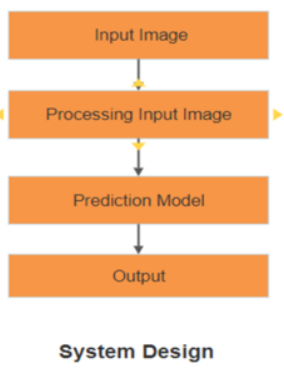

Figure.3 System Design

Input Image: Here we feed the image as an input by capturing the image of the food using our app.Processing Input Image: Before sending the picture to the Prediction model the image must be processed.In this processing phase the image will be cropped and only the required features will be extracted for prediction.Prediction Model:Processed image is fed into prediction model. This model will classify the image of the food fed to it by analysing the patterns of the image.Output: After this complete process the output will be given on the screen. This output can the name of 
the food, recipe of the food.

\section{Methodology}

Data Collection: We gather food pictures by taking image or via looking through web images and fabricate a picture dataset index to use in preparing a complex recognition model for food things. Data Cleaning: This is to recognize and eliminate any possible mistakes and copy information, to make a reliable dataset. This improves the idea of the training data for investigation and enables exact decision making. Training Data Set: This is the real dataset that we will use to train the model. The model will analyze and gains from this information gave. Validation Data Set: The approval collection is primarily used to assess the given framework, yet this is for frequent assessment. We utilize this information to fine-tuning the model hyper parameters. Consequently, the model occasionally investigates this information, yet it won't ever gain from this. We utilize the validation outcomes and update to more higher level hyper parameters. Along these lines, the validation set will influence the model, yet just in indirect way. The validation set is otherwise called the set of development. This makes sense the dataset helps during the "advancement" phase of the model. Testing Data Set: The Test dataset is just utilized once the model is totally prepared by utilizing the train and validation sets for the model. The test set is essentially what is utilized to assess the competing models. Large numbers of the occasions the validation set is utilized as a test set, yet it's anything but a decent practice. The test set is for the most part in the administration. It contains deliberately accumulated information that navigates the different classes of model would confront when it is taken truly.

\section{Expected output and Result}

After completion of this project, a lens app is created for smartphones where a box is displayed on the phone screen. So, users can scan by pointing the lens towards the food and tapping on the screen. Then the application will recognize the food, after which it will show the name of the food item, its recipe and nutrition contents.

\section{Applications}

1. Useful for diabetic and other patients to maintain their dietary plans.

2. When a user visits any hotel, this app helps them know the recipe of the food they have ordered.

3. For users who want to maintain a healthy and fit body, this app helps them by providing Appropriate nutrition contents in the food.

\section{Conclusion}

The food culture is a growing rapidly and the accurate access to data is very much required more than ever, we propose to solve these problems and provide user with the correct data they need which will make user to not spend more time to search for the data they need, rather they can simply scan the food and get all the information. 


\section{References}

[1] BahmanJavadi, Quoc Lap Trieu, Kenan M. Matawie,Rodrigon.Calheiros. Smart Food Scanner BasedOn Mobile Edge Computing . IEEE International Conference On Cloud Engineering (Ic2e), Page No. 20-27, May- 25,2020.

[2] Weiransong,Hui Wang, Jiangnanfeng, Use Of Smartphone Videos Andpattern Recognition For Food Authentication, Sensors And Actuators Bchemical,Researchgate,June-05,2020.

[3] Avinashpalve, Snehal More, ShivaniChaudhari, Akshaykatke, Karthiekampassi, An Android Based Health Monitoring System Using Machine Learning, Irjet, Volume:03, Sep-2016.

[4] Yoshiyuki Kawano, Keijiyanai, Real-Time Mobile Food Recognition System,2013.

[5] GianluigiCiocca,Paolonapoletano,RaimondoSchettini, Food Recognition: A New Dataset, Experiments, And Results ,Ieee Journal Of Biomedical And Healthinformatics, Volume: 21,May2017.

[6] Ayush Jain, MohanaMohana, Real Time Object Detection And Tracking Using Deep Learning And Opencv, July 2018.

[7] S.Hema Kumar, J.UdayKiran, V.D.Akumar, G.Saranya, Ramalakshmi V.Effective Online Medical Appointment System. International Journal Of Scientific \& Technology Research, Volume 8, Issue 09, September 2019, Pages 803 - 805 .

[8] Ambeth Kumar.V.D, Dr.M.Ramakrishnan, V.D.Ashok Kumar And Dr.S.Malathi (2015) .Performance Improvement Using An Automation System For Recognition Of Multiple Parametric Features Based On Human Footprint. For The International Journal Of Kuwait Journal Of Science \& Engineering, Vol 42, No 1 (2015), Pp:109-132.

[9] V.D.Ambeth Kumar, V.D.Ashok Kumar, S.Malathi, K.Vengatesan, M.Ramakrishnan. Facial Recognition System For Suspect Identification Using A Surveillance Camera.Pattern Recognition And Image Analysis (Springer), Volume 28, Issue 3, Pp 410-420, $2018 . \quad$ (Doi: 10.1134/S1054661818030136)

[10] V.D.Ambeth Kumar And M.Ramakrishan, "Employment Of Footprint Recognition System", In The Month Of December For Indian Journal Of Computer Science And Engineering (Ijcse) Vol. 3 No.6 Dec 2013

[11] Ramya,T.,Dr.Malathi,S.,Ratheeksha,G.R. And Dr.V.D.Ambeth Kumar (2014). Personalized Authentication Procedure For Restricted Web Service Access In Mobile Phones. Applications Of Digital Information And Web Technologies (Icadiwt), 2014, Page(S):69 - 74, Bangalore, India (Isbn:978-1-4799-2258-1)

[12] Ambeth Kumar.V.D (2018). Novel Wireless Sensing System For The Welfare Of Sewer Laborers. Healthcare Technology Letters (Iet) Volume 5, Issue 4, P. $107-112$.

[13] Ambeth Kumar.V.D, G.Saranya , D.Elangovan, V.Rahulchiranjeevi, V.D.Ashok Kumar.’Iot Based Smart Museum Using Wearable Device". Lecture Notes In Networks And Systems, Vol.55, Pp: 33-42, 2018

[14] Ambeth Kumar.V.D And Dr.M.Ramakrishan,'Footprint Based Recognition System", Communication In Computer And Information System, Volume 147, Part 3, 358-367, Doi: 10.1007/978-3-642-205736_63, April 2011

[15] Ambeth Kumar.V.D (2017).Efficient Routing For Low Rate Wireless Network A Novel Approach. International Journal Of Image Mining, Vol. 2, Nos. 3/4, 2017, 2017

[16] Ambeth Kumar.V.D, V.D.Ashok Kumar, Dr.S.MalathiAndP.Jagaeedesh, (2014) .Intruder Identification Using Footprint Recognition With Pca And Svm Classifiers. For The International Journal Of Advanced Materials Research Vols.1345, Pp 984-985 (2014) Pp 1345-1349. [Doi:10.4028/Www.Scientific.Net/Amr.984-985.1345]

[17] B. Aravindh; V.D.Ambeth Kumar; G. Harish; V. Siddartth, " A novel graphical authentication system for secure banking systems”, IEEE (ICSTM), Pages: $177-183$, 2-4 Aug. 2017, DOI: 10.1109/ICSTM.2017.8089147.

[18] R. Subha Shini et.al., “ Recurrent Neural Network based Text Summarization Techniques by Word Sequence Generation",IEEE International Conference on Inventive Computation Technologies (ICICT), 2021, DOI: 10.1109/ICICT50816.2021.9358764 